OPEN ACCESS

Edited by:

Michael Gänzle,

University of Alberta, Canada

Reviewed by:

Vijay K. Juneja,

United States Department

of Agriculture, United States

Magaly Toro,

Universidad de Chile, Chile

*Correspondence: Qingping Wu wuqp203@163.com; wuqp@gdim.cn

${ }^{\dagger}$ These authors have contributed equally to this work.

Specialty section: This article was submitted to

Food Microbiology,

a section of the journal

Frontiers in Microbiology

Received: 24 October 2017 Accepted: 14 May 2018

Published: 05 June 2018

Citation:

Ling N, Li C, Zhang J, Wu Q, Zeng $H$, He W, Ye $Y$, Wang J,

Ding $Y$, Chen $M, X u e ~ L, Y e Q$ and

Guo W (2018) Prevalence and Molecular and Antimicrobial Characteristics of Cronobacter spp. Isolated From Raw Vegetables in China. Front. Microbiol. 9:1149. doi: 10.3389/fmicb.2018.01149

\section{Prevalence and Molecular and Antimicrobial Characteristics of Cronobacter spp. Isolated From Raw Vegetables in China}

\author{
$\mathrm{Na}$ Ling ${ }^{1,2+}$, Chengsi $\mathrm{Li}^{2+}$, Jumei Zhang ${ }^{2+}$, Qingping Wu ${ }^{2 *}$, Haiyan Zeng ${ }^{2}$, Wenjing $\mathrm{He}^{2}$, \\ Yingwang Ye², Juan Wang ${ }^{3}$, Yu Ding ${ }^{4}$, Moutong Chen², Liang Xue'2, Qinghua $\mathrm{Ye}^{2}$ and \\ Weipeng $\mathrm{Guo}^{2}$
}

${ }^{1}$ School of Bioscience and Bioengineering, South China University of Technology, Guangzhou, China, ${ }^{2}$ State Key Laboratory of Applied Microbiology, South China, Guangdong Provincial Key Laboratory of Microbiology Culture Collection and Application, Guangdong Open Laboratory of Applied Microbiology, Guangdong Institute of Microbiology, Guangzhou, China, ${ }^{3}$ College of Food Science, South China Agricultural University, Guangzhou, China, ${ }^{4}$ Department of Food Science \& Technology, Jinan University, Guangzhou, China

Cronobacter spp. is a foodborne pathogen that causes life-threatening and invasive diseases, such as necrotizing enterocolitis, meningitis, and sepsis. In this study, we aimed to investigate the prevalence, molecular characteristics and antimicrobial resistance of Cronobacter spp. in raw vegetables marketed in China. Based on dietary habits in China, 403 raw vegetables that could be eaten without additional cooking were collected. Of the 403 samples tested, 122 (30.27\%) were positive for Cronobacter spp., and the contamination levels exceeded 110 most probable number (MPN)/g for $16.39 \%(20 / 122)$ of the samples. Coriander samples had the highest contamination rate of $52.81 \%$, and the MPN values of $19.15 \%$ of positive coriander samples exceeded $100 \mathrm{MPN} / \mathrm{g}$. Eleven serotypes were identified among 171 isolates, with Cronobacter sakazakii serogroup $\mathrm{O} 1$ (41 isolates) being the dominant serotype. Molecular characterization indicated that there was quite high genetic diversity in Cronobacter spp., and multilocus sequence typing analyses yielded 106 sequence types (STs), 55 of which were newly identified. Notably, the most prevalent ST (eight isolates) was C. malonaticus ST60, which appeared in a recent clinical infectious disease study in China. Five C. sakazakii ST4, seven C. malonaticus ST7, and three C. sakazakii ST8 confirmed as pathogenic STs in other countries were also detected in this study. Furthermore, all isolates were susceptible to amikacin, amoxicillin-clavulanic, cefepime, ciprofloxacin, and imipenem, but some isolates exhibited a high ratio of resistance to cephalothin (59.65\%). In this study, the high contamination rate and the detection of pathogenic and new STs in raw vegetables indicated potential hazards to customers. To the best of our knowledge, this is the first report to provide valuable information on the contamination status of Cronobacter spp. in vegetables that can be eaten raw in China.

Keywords: Cronobacter spp., vegetable, multilocus sequence typing, serotype, antimicrobial sensitivity 


\section{INTRODUCTION}

The genus Cronobacter comprises opportunistic pathogens that can cause life-threatening infection in neonates and infants with necrotizing enterocolitis, bacteraemia and meningitis with case fatality rates ranging between 40 and $80 \%$ (Sulovska et al., 2017). In addition, infections of Cronobacter also occur in older children and adults. In adults, the majority of Cronobacter infections are the elderly population, especially those suffering from serious underlying disease or malignancy. In adults, Cronobacter can lead to urinary tract infections, septicemia, pneumonia, osteomyelitis, wound infections, and splenic abscesses (Patrick et al., 2014; Alsonosi et al., 2015). The Cronobacter genus consists of seven species: C. sakazakii, C. malonaticus, C. turicensis, C. muytjensii, C. dublinensis, C. universalis, and C. condimenti (Iversen et al., 2008; Joseph et al., 2012a). Strains belonging to C. sakazakii, C. malonaticus, and C. turicensis can cause infections in humans (Joseph et al., 2012c). However, the inability of many clinical laboratories to identify Cronobacter in a timely manner may allow many infections to go undiscovered (Holy and Forsythe, 2014).

Cronobacter spp. have been detected in a wide spectrum of foods, including dairy, cereals, flour, medicinal plants, herbs and spices, rice, meat, fruits, vegetables, and macro fungi, as well as their by-products (Iversen and Forsythe, 2003; Molloy et al., 2009; Cetinkaya et al., 2013; Ye et al., 2014a). The plant environment is considered to be the natural habitat of Cronobacter (Schmid et al., 2009). Therefore, non-infant populations could be affected by foods of plant origin contaminated with Cronobacter because vegetables are a major part of the normal diet; however, no clear epidemiological link has been established yet between the consumption of raw vegetables and diseases caused by Cronobacter spp. Accordingly, more attention should be paid to the food matrices of plant materials due to frequent contamination (Singh et al., 2015). The use of lettuces, corianders, cucumbers, and tomatoes as the main ingredients in ready-toeat food, such as green salad, is common throughout the world. These four kinds of raw vegetables selected in this study are of great significance, as they are prevalently eaten without cooking, heating or cooling, depending on habitual meal patterns in China. Importantly, outbreaks of Escherichia coli and Salmonella have been shown to be associated with consumption of lettuce and cucumbers, and these vegetables may represent a major threat to human health (Nygård et al., 2008; Söderström et al., 2008; Zhang et al., 2009; Tuffs, 2011; Taylor et al., 2013; Angelo et al., 2015). However, limited data are available regarding the presence of Cronobacter spp. in raw vegetables in China. Therefore, a risk forecast evaluating the contamination status, molecular affiliations, source, and related drug-resistance of Cronobacter in vegetables that can be eaten raw is necessary.

Understanding the genetic diversity of Cronobacter can contribute to accurate identification at the genus and species levels and may facilitate reliable source tracking of contaminated foods. To date, Cronobacter isolates can be further characterized by a range of molecular typing methods (Ye et al., 2014b). $\mathrm{O}$-antigen variations are of great significance in epidemiological studies (Blazkova et al., 2015). Based on O-antigens, 17 $O$-serogroups of the genus Cronobacter have been identified
(Mullane et al., 2008; Jarvis et al., 2011, 2013; Sun et al., 2012b). In our previous study, the enterobacterial repetitive intergenic consensus sequence polymerase chain reaction (ERIC-PCR) fingerprint was applied as a serviceable tool for the molecular characterization and identification of Cronobacter strains (Ye et al., 2009; Ye et al., 2010). Furthermore, multilocus sequence typing (MLST) based on seven housekeeping genes has proven to be a more robust means of identifying and discriminating species of the Cronobacter genus than biotyping, such as $16 \mathrm{~S}$ rRNA gene sequence analysis, ERIC-PCR and pulsed-field gel electrophoresis (Baldwin et al., 2009; Joseph et al., 2012c). The Cronobacter PubMLST database is available online at http:// pubmlst.org/cronobacter/. Furthermore, several sequence types (STs) have been shown to be associated with outbreaks (Joseph and Forsythe, 2012). Notably, C. sakazakii clonal complex 4 was found to be the predominant ST in clinical sources and appeared in the majority of neonatal meningitis cases (Forsythe et al., 2014).

Currently, antibiotic therapy is the clinically preferred and most widespread method for the treatment of Cronobacter infection (Depardieu et al., 2007). However, prolonged and extensive use of antibiotics can accelerate bacterial resistance to antimicrobial agents. Recently, Cronobacter spp. have been reported to exhibit resistances to amoxicillin-clavulanate, ampicillin, cefazolin, penicillin G, and streptomycin in some isolates (Lee et al., 2012; Xu et al., 2015; Fei et al., 2017). The discovery of the colistin-resistance gene mcr-1 in Cronobacter has highlighted the importance of antibiotic resistance in this organism (Liu et al., 2017). Therefore, screening Cronobacter isolates from food for antibiotic resistance could be useful in association analysis with clinical isolates and could be of significance to public health and environmental pollution studies.

Cronobacter are considered a plant-associated organism and may therefore be highly prevalent in raw vegetables. Therefore, in this study, we aimed to characterize the prevalence of Cronobacter spp. in raw vegetables from Chinese markets using phenotyping and genotyping methods in order to determine the genetic relatedness among isolates. We focused on determining the contamination levels, serological types, MLST types, and antibiotic resistance patterns of the isolated strains to characterize the diversity of Cronobacter spp. in vegetables from Chinese markets.

\section{MATERIALS AND METHODS}

\section{Sampling}

A total of 403 vegetable samples (lettuce $=87$, coriander $=89$, tomato $=104$, cucumber $=123$ ) were collected from July 2011 to July 2016 in 39 cities in China and tested for the presence of Cronobacter isolates (Table 1). The sampling sites covered most of the provincial capitals of China, and sites chosen have the certain representation on region. China was divided to seven geographical regions based on the Chinese regional strategy for the topography, climate, humanity, economy and policy of the Chinese government (Figure 1). In each city, approximately 10 samples were 
TABLE 1 | Prevalence and level of Cronobacter spp. in different foods.

\begin{tabular}{|c|c|c|c|c|}
\hline \multirow[t]{2}{*}{ Food } & \multirow[t]{2}{*}{ Prevalence rate $(\%)$} & \multicolumn{3}{|c|}{ MPN value (MPN/g) } \\
\hline & & MPN < $10(\%)$ & $10 \leq \mathrm{MPN}<110(\%)$ & $110 \leq$ MPN (\%) \\
\hline Lettuce & 26/87 (29.89) & $17 / 26(65.38)$ & $3 / 26(11.54)$ & $6 / 26(23.08)$ \\
\hline Coriander & $47 / 89(52.81)$ & $30 / 47$ (63.83) & $8 / 47(17.02)$ & $9 / 47(19.15)$ \\
\hline Tomato & 13/104 (12.50) & 11/13 (84.62) & $0 / 13(0)$ & $2 / 13$ (15.38) \\
\hline Cucumber & $36 / 123$ (29.27) & 30/36 (83.33) & 3/36 (8.33) & 3/36 (8.33) \\
\hline Total & 122/403 (30.27) & 88/122 (72.13) & 14/122 (11.78) & 20/122(16.39) \\
\hline
\end{tabular}

collected from two supermarkets and two traditional retail markets and supermarkets. Details of sample distribution are shown in Supplementary Table S1. Food samples were transferred under cold conditions (below $4^{\circ} \mathrm{C}$ ) to the laboratory and were immediately subjected to microbiological analysis. Bioexperiments were operated in class II biosafety cabinets in laboratory of BSL2.

\section{Detection of Cronobacter spp. Strains}

An enrichment method was performed according to the National Food Safety Standard of China for food microbiological examination of Enterobacter sakazakii (GB 4789.40-2010, National Standard of the People's Republic of China, 2010), with minor modifications (Xu et al., 2015). Briefly, $25 \mathrm{~g}$ of sample was homogenized for $60 \mathrm{~s}$ in stomacher bags (Huankai, Guangzhou, China) with $225 \mathrm{~mL}$ of modified lauryl sulfate tryptose broth-vancomycin medium (mLST-Vm), in which vancomycin was added at a final concentration of $10 \mu \mathrm{g} / \mathrm{mL}$. A nine-tube method was adopted to determine the most probable number (MPN). The nine tubes were divided into three sets of three tubes each. The second and third sets of tubes contained 10 $\mathrm{mL}$ of mLST. Three aliquots $(10,1$, and $0.1 \mathrm{~mL})$ of the sample homogenate were dispensed into the three sets, representing $1.0,0.1$, and $0.01 \mathrm{~g}$ of the original sample, respectively. Nine tubes were incubated at $44^{\circ} \mathrm{C}$ overnight and were then streaked onto chromogenic medium and incubated at $44^{\circ} \mathrm{C}$ for $24 \mathrm{~h}$. Presumptive Cronobacter spp. presenting green or blue-green colonies were selected for analysis using API 20E diagnostic strips (BioMérieux, Marcy-l'Étoile, France) and species identification was performed by fusA sequencing (Joseph et al., 2012c). MPN was determined on the basis of the number of positive tube(s) in each of the three sets and the MPN table (GB 4789.7-2013,

\section{China Regional Strategy}

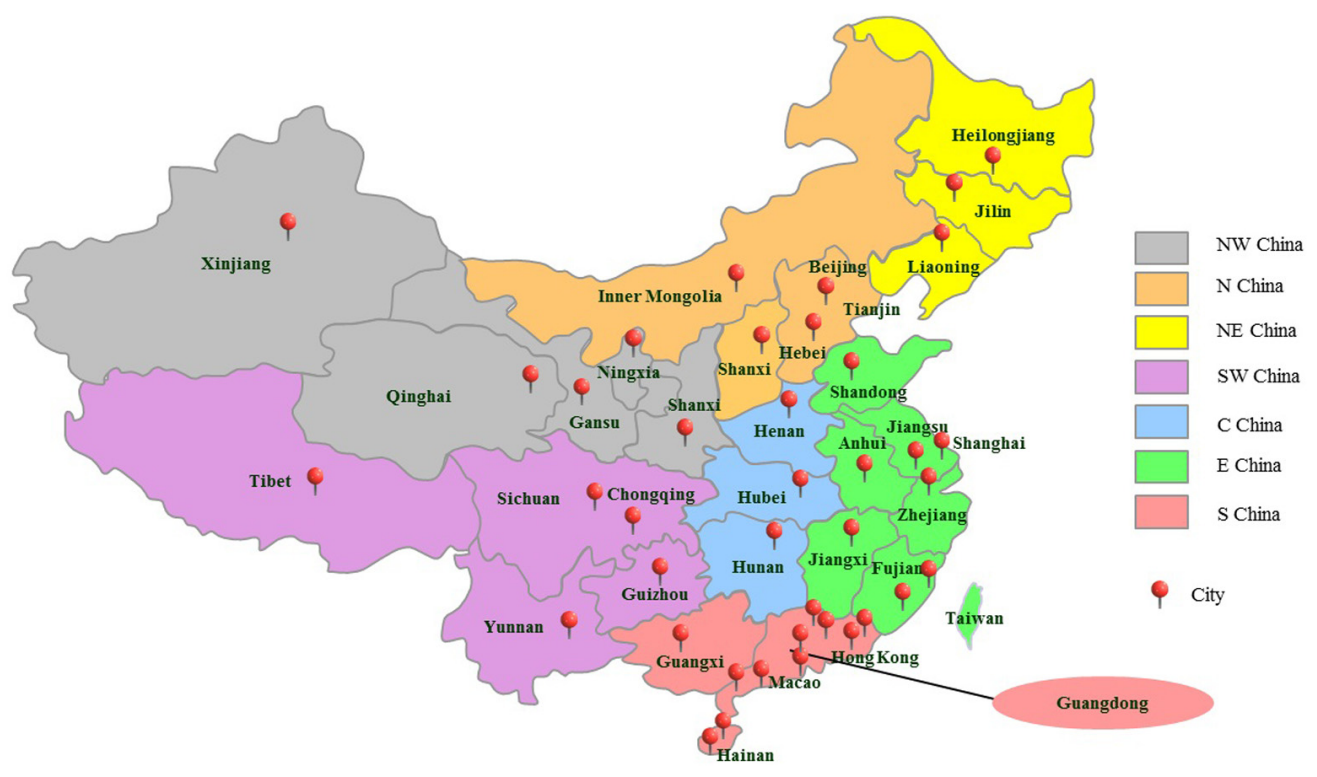

FIGURE 1 | The locations of the sampling sites for this study in China, including 39 cities. Seven geographical divisions based on the Chinese regional strategy are indicated using different colors. E, east; N, north; S, south; NE, northeast; NW, northwest. SW, southwest; C, central. 
National Standard of the People's Republic of China, 2013; Xu et al., 2015).

\section{Molecular Serotyping Identification of Cronobacter spp.}

The detection of Cronobacter spp. serotypes was performed by PCR. Overall, 14 serotypes were identified using protocols and primers according to previously reported Cronobacter molecular serotyping schemes, including C. sakazakii O:1 to O:7 (Jarvis et al., 2011; Sun et al., 2011, 2012b), C. turicensis O:1 to O:3, C. malonaticus $\mathrm{O}: 1$ and $\mathrm{O}: 2$, and $C$. dublinensis $\mathrm{O}: 1$ and $\mathrm{O}: 2$ (Jarvis et al., 2011, 2013).

\section{MLST and Sequence Analysis}

Multilocus sequence typing was applied to Cronobacter spp. molecular typing according to a previous study reported by Joseph et al. (2012b). Seven housekeeping genes were amplified with primers and conditions according to a protocol for Cronobacter available at the MLST web database ${ }^{1}$. Comparing the seven loci sequences with this database generated the allele codes and STs of the strains, and the designation of new alleles and STs was verified by the MLST database curator. A neighbor-joining phylogenetic tree was generated based on the seven MLST loci (3,036 bp concatenated length) of the Cronobacter spp. strains. This tree was generated using MEGA 5 (version 5.05) with 1000 bootstrap replicates. A minimum spanning tree was constructed in BioNumerics software (Applied Maths, Sint-Martens-Latem, Belgium) according to relationships among MLST alleles.

\section{Antimicrobial (AM) Susceptibility Test}

The susceptibility profile of Cronobacter isolates was determined by the standard disk diffusion method using Mueller-Hinton agar (Huankai) following the guidelines of the (Clinical and Laboratory Standards Institute [CLSI], 2006). The following 16 antibiotics (Oxoid, Hampshire, United Kingdom) were classified into nine different groups (Table 5) according to the World Health Organization [WHO] (2007): ampicillin (AMP, $10 \mu \mathrm{g}$ ), ampicillin/sulbactam (SAM, $10 \mu \mathrm{g} / 10 \mu \mathrm{g}$ ), amoxicillin/clavulanic acid (AMC, $20 \mu \mathrm{g} / 10 \mu \mathrm{g}$ ), cefazolin (KZ, $30 \mu \mathrm{g}$ ), cephalothin (KF, $30 \mu \mathrm{g}$ ), cefepime (FEP, $30 \mu \mathrm{g}$ ), ceftriaxone (CRO, $30 \mu \mathrm{g})$, gentamicin (CN, $10 \mu \mathrm{g})$, tobramycin (TOB, $10 \mu \mathrm{g}$ ), amikacin (AK, $30 \mu \mathrm{g}$ ), chloramphenicol (C, $30 \mu \mathrm{g}$ ), ciprofloxacin (CIP, $5 \mu \mathrm{g}$ ), imipenem (IPM, $10 \mu \mathrm{g}$ ), trimethoprim/sulfamethoxazole (SXT, $1.25 \mu \mathrm{g} / 23.75 \mu \mathrm{g}$ ), aztreonam (ATM, $30 \mu \mathrm{g}$ ), and tetracycline (TE, $30 \mu \mathrm{g}$ ). E. coli ATCC 25922 was used as a quality control organism.

\section{Statistical Analysis}

Statistical analysis was performed using the SPSS (version 18.0, SPSS, Inc, Chicago, IL, United States) software package. For prevalence data, the chi-square and the Fisher exact test were used for comparison of prevalence rates among the sample sites and prevalence of Cronobacter spp. in four vegetables. $P<0.05$ was considered significant.

${ }^{1}$ http://pubmlst.org/cronobacter/

\section{RESULTS}

\section{Prevalence of Cronobacter spp. in Non-heat Treated Vegetables}

Of the 403 vegetable samples, 122 were confirmed to contain Cronobacter spp. (Table 1). The prevalence rate of Cronobacter varied among the different foods, as follows: $29.89 \%(26 / 87)$ in lettuce, $52.81 \%(47 / 89)$ in coriander, $12.50 \%(13 / 104)$ in tomato, and $29.27 \%(36 / 123)$ in cucumber (Table 1). Based on quantitative analysis, 53 of the positive samples (43.44\%) were contaminated at a level between 0.3 and $10 \mathrm{MPN} / \mathrm{g}$; contamination levels exceeded $110 \mathrm{MPN} / \mathrm{g}$ in 20 samples. Isolates from the same sample belonging to the same ST were considered clonal. Thus, 171 Cronobacter spp. isolates were isolated from the 122 positive samples for further study. Of the 171 recovered isolates, the following species were identified: 89 C. sakazakii (52.04\%), 36 C. dublinensis (21.05\%), 39 C. malonaticus (22.81\%), and seven $C$. turicensis isolates (4.10\%). The prevalence of Cronobacter spp. varied among the seven geographical divisions of China, ranging from 22.45\% in Northwest China to $35.21 \%$ in South China (Table 2). Notably, C. dublinensis seemed to be present most often in Central China $(P<0.01)$ (Table 3).

\section{Serogroup Analysis}

PCR-based $\mathrm{O}$-antigen serotyping techniques were applied to obtain data on the distribution of $O$-antigen serotypes among the 171 Cronobacter spp. isolates. The occurrence of Cronobacter spp. in the samples is summarized in Table 4. The serotypes O:5 and O:6 were not detected in C. sakazakii isolates. C. sakazakii serotype O:1 was the dominant serotype (41 strains), followed by serotype O:2 (31 strains). Thirty-nine C. malonaticus strains were classified into two serotypes (O:1 and O:2), with the serotype O:1 covering 20 isolates. Nineteen $C$. dublinensis $O: 1$ and 11 C. malonaticus O:2 isolates were identified. Some strains were identified as C.turicensis O:1 and O:3.

\section{Antimicrobial Susceptibility Test}

The 171 Cronobacter spp. isolates were subjected to 16 antimicrobial susceptibility tests, and the results are shown in Table 5. All examined isolates were susceptible to $\mathrm{CN}, \mathrm{AK}$, SAM, FEP, CIP, IPM, C, AMC, and ATM. The susceptibility tests revealed that the isolates exhibited relatively high resistance

TABLE 2 | The contamination situation of Cronobacter spp. in seven geographical divisions of China.

\begin{tabular}{lcccc}
\hline Region & $\begin{array}{c}\text { Positive } \\
\text { samples }\end{array}$ & $\begin{array}{c}\text { Negative } \\
\text { samples }\end{array}$ & $\begin{array}{c}\text { Total } \\
\text { samples }\end{array}$ & $\begin{array}{c}\text { Prevalence } \\
\text { rate (\%) }\end{array}$ \\
\hline S China & 50 & 92 & 142 & $35.21 \%$ \\
E China & 25 & 52 & 77 & $32.47 \%$ \\
C China & 8 & 21 & 29 & $27.59 \%$ \\
N China & 11 & 27 & 38 & $28.95 \%$ \\
NE China & 7 & 23 & 30 & $23.33 \%$ \\
NW China & 11 & 38 & 49 & $22.45 \%$ \\
SW China & 10 & 28 & 38 & $26.32 \%$ \\
Total & 122 & 281 & 403 & $30.27 \%$
\end{tabular}


TABLE 3 | The distribution of Cronobacter species in seven geographical divisions of China.

\begin{tabular}{|c|c|c|c|c|}
\hline Region & C. sakazakii & C. malonaticus & C. dublinensis & C. turicensis \\
\hline S China & $37 / 66$ (56.06\%) & 18/66 (27.27\%) & $9 / 66$ (13.64\%) & 2/66 (3.03\%) \\
\hline E China & $22 / 45$ (48.89\%) & $14 / 45$ (31.11\%) & $7 / 45$ (15.55\%) & $2 / 45$ (4.44\%) \\
\hline C China & $1 / 13(7.69 \%)$ & $1 / 13(7.69 \%)$ & $11 / 13(84.61 \%)$ & 0/13 (0.00\%) \\
\hline N China & 10/15 (66.67\%) & $3 / 15$ (20.00\%) & 2/15 (13.33\%) & 0/15 (0.00\%) \\
\hline NE China & $5 / 7(71.43 \%)$ & 0/7 (0.00\%) & $1 / 7(14.28 \%)$ & $1 / 7$ (14.28\%) \\
\hline NW China & 10/13 (76.92\%) & 1/13 (7.69\%) & 1/13 (7.69\%) & 1/13 (7.69\%) \\
\hline SW China & 4/12 (33.33\%) & $2 / 12$ (16.67\%) & $5 / 12$ (41.67\%) & $1 / 12$ (8.33\%) \\
\hline
\end{tabular}

TABLE 4 | Species and serotypes of Cronobacter spp. isolates in this study.

\begin{tabular}{|c|c|c|}
\hline Species & Serotype & No. of isolates \\
\hline \multirow[t]{5}{*}{ C. sakazakii } & O:1 & 41 \\
\hline & $0: 2$ & 31 \\
\hline & $0: 3$ & 6 \\
\hline & $0: 4$ & 5 \\
\hline & $0: 7$ & 6 \\
\hline \multirow[t]{3}{*}{ C. dublinensis } & O:1 & 19 \\
\hline & $0: 2$ & 11 \\
\hline & Uncertain & 6 \\
\hline \multirow[t]{3}{*}{ C. malonaticus } & $0: 1$ & 20 \\
\hline & $0: 2$ & 16 \\
\hline & Uncertain & 3 \\
\hline \multirow[t]{3}{*}{ C. turicensis } & O:1 & 1 \\
\hline & $0: 3$ & 4 \\
\hline & Uncertain & 2 \\
\hline Total & & 171 \\
\hline
\end{tabular}

The detection of Cronobacter spp. serotypes was performed by PCR according to Jarvis et al. (2011, 2013) and Sun et al. (2012a; 2012b).

to $\mathrm{KF}$, with resistance and intermediate rates of 59.65 and $38.60 \%$, respectively. Among the remaining tested antibiotics, the next-highest intermediate resistance rate was observed for $\mathrm{KZ}$ (28.65\%). Two isolates exhibited resistance to SXT, and three strains showed resistance to TE. However, the two isolates were multidrug-resistant strains that showed resistance to three AMs, i.e., KF, SXT, and TE.

\section{MLST Sequence Analysis of Cronobacter spp. Isolates}

The phylogenetic tree generated based on the concatenated sequences of seven loci (Figure 2) showed clear clustering across the Cronobacter spp.; consequently, the 171 isolates were sorted into four species. BioNumerics (version 7.6) was used to construct a phylogenetic dendrogram to estimate relationships among isolates. Overall, 171 isolates were assigned to 106 STs, of which 55 were novel (Supplementary Table S2). Among these 106 STs, 79 of the 106 STs were represented only by one strain, and the remaining 27 STs covered $2-8$ isolates. ST60 was the dominant ST $(n=8)$, followed by ST7 and ST64, which each included seven strains. ST4, ST23, ST148, and ST211, which were associated with clinical isolates, included five isolates. Furthermore, ST4, ST64, and ST7 were found in all four vegetables; ST17, ST23, ST37, and
ST211 were found in coriander, lettuce, and cucumber; ST77 was found in coriander, cucumber, and tomato; ST13 was found in coriander, lettuce, and tomato; and ST60 was only detected in coriander and cucumber (Figure 3).

\section{DISCUSSION}

Cronobacter spp. are responsible for fatal infections caused by ingestion of contaminated infant formula (Van Acker et al., 2001; Stoll et al., 2004; Li et al., 2016). Additionally, Cronobacter isolates may be present in a diverse range of environments and in foods that are commonly consumed. Because China is a large agricultural country with relatively abundant resources, considerable attention should be paid to vegetable safety issues related to microorganisms. To date, limited data are available regarding Cronobacter spp. present in raw vegetables.

In this study, owing to common Chinese dietary habits, 403 raw vegetables that could be eaten without additional cooking were examined. Overall, the prevalence of Cronobacter spp. in vegetables was determined to be $30.27 \%$; contamination levels of Cronobacter spp. exceeded $110 \mathrm{MPN} / \mathrm{g}$ in 20 samples. Notably, coriander samples had the highest contamination rate of $52.81 \%$ $(P<0.01)$, and the MPN value of $19.15 \%$ of positive coriander samples exceeded $100 \mathrm{MPN} / \mathrm{g}$. However, the methodology used in this study has not been standardized for vegetables, and it was developed and standardized for detecting Cronobacter spp. from powdered infant formula; thus, the isolation rates may have been underestimated. Several previous studies have reported the occurrence of Cronobacter strains in vegetables. For example, Chon et al. (2012) and Lee et al. (2012) isolated Cronobacter spp. in $14.8 \%$ and $30 \%$ of vegetables in Korea, respectively. In addition, a study on the prevalence of this bacteria in plant-based food and related environments was undertaken in the Czech Republic and detected Cronobacter spp. in $8.0 \%$ of samples (Vojkovska et al., 2016). Recently, 15 of 102 vegetable samples were found to be positive in the middle-east coastline of China (Chen et al., 2016). Furthermore, in our previous study, samples of cold vegetables in sauce were contaminated with Cronobacter spp. at a particularly high rate of $41.9 \%$ ( $\mathrm{Xu}$ et al., 2015). This great variability observed in different studies is probably due to the number and species of samples analyzed and cross-contamination resulting from open vegetable display areas. In addition, the prevalence of Cronobacter spp. varied in seven geographical divisions of China, 
TABLE 5 | Antimicrobial resistance profiles of 171 Cronobacter spp. isolates.

\begin{tabular}{|c|c|c|c|c|c|c|}
\hline \multirow[t]{2}{*}{ Antimicrobial group } & \multirow[t]{2}{*}{ Antibiotic } & \multirow[t]{2}{*}{ Disk code } & \multirow{2}{*}{$\begin{array}{l}\text { Antimicrobial class } \\
\text { according to the WHO }\end{array}$} & \multicolumn{3}{|c|}{ No. $(\%)$ of Cronobacter spp. $(n=171)$} \\
\hline & & & & Resistant & Intermediate & Susceptible \\
\hline \multirow[t]{3}{*}{ Penicillins } & Ampicillin & AMP & $\mathrm{Cl}$ & $0(0)$ & $1(0.58)$ & $170(99.42)$ \\
\hline & Ampicillin/sulbactam & SAM & $\mathrm{Cl}$ & $0(0)$ & $0(0)$ & $171(100)$ \\
\hline & Amoxicillin/clavulanic & AMC & $\mathrm{Cl}$ & $0(0)$ & $0(0)$ & $171(100)$ \\
\hline \multirow[t]{4}{*}{ Cephalosporins } & Cefepime & FEP & $\mathrm{Cl}$ & $0(0)$ & $0(0)$ & $171(100)$ \\
\hline & Ceftriaxone & $\mathrm{CRO}$ & $\mathrm{Cl}$ & $0(0)$ & $4(2.34)$ & $167(97.66)$ \\
\hline & Cefazolin & $\mathrm{KZ}$ & $\mathrm{HI}$ & $0(0)$ & $49(28.65)$ & $122(71.35)$ \\
\hline & Cephalothin & $\mathrm{KF}$ & $\mathrm{HI}$ & 102 (59.65) & $66(38.60)$ & $3(1.75)$ \\
\hline \multirow[t]{3}{*}{ Aminoglycosides } & Gentamicin & $\mathrm{CN}$ & $\mathrm{Cl}$ & $0(0)$ & $0(0)$ & $171(100)$ \\
\hline & Tobramycin & TOB & $\mathrm{Cl}$ & $0(0)$ & $3(1.75)$ & $168(98.25)$ \\
\hline & Amikacin & AK & $\mathrm{Cl}$ & $0(0)$ & $0(0)$ & $171(100)$ \\
\hline Quinolones & Ciprofloxacin & $\mathrm{CIP}$ & $\mathrm{Cl}$ & $0(0)$ & $0(0)$ & $171(100)$ \\
\hline Carbapenems & Imipenem & IPM & $\mathrm{Cl}$ & $0(0)$ & $0(0)$ & $171(100)$ \\
\hline Sulfonamides & Trimethoprim/sulfamethoxazole & SXT & $\mathrm{HI}$ & $2(1.17)$ & $0(0)$ & 169 (98.83) \\
\hline Monobactams & Aztreonam & ATM & $\mathrm{HI}$ & $0(0)$ & $0(0)$ & $171(100)$ \\
\hline Amphenicols & Chloramphenicol & C & $\mathrm{HI}$ & $0(0)$ & $0(0)$ & $171(100)$ \\
\hline Tetracyclines & Tetracycline & TE & $\mathrm{HI}$ & $3(1.75)$ & $0(0)$ & 168 (98.25) \\
\hline
\end{tabular}

aCl, critically important; HI, highly important; I, important.

ranging from $22.45 \%$ in Northwest China to $36.11 \%$ in South China. Surprisingly, the presence of C. dublinensis in Central China was significantly higher than those in other regions $(P<0.01)$; however, the exact reason for this phenomenon is still unknown. Because the sample size was not large enough, continuous surveillance of Cronobacter in China for reliable source tracking and determination of the contamination status is necessary. Additionally, no standards for limitation of Cronobacter spp. in vegetables have been established in China. Lettuce and cucumbers caused the life-threatening infections owing to E. coli and Salmonella contamination. Cases of human Cronobacter infection have not been linked to its presence in raw vegetables, however, raw vegetables may potentially disperse Cronobacter and may contribute to human infections. Particularly immune-compromised individuals, the elderly and hospitalized patients may be at risk when eating raw vegetables without heating.

In this study, 171 Cronobacter spp. isolates were isolated from 122 positive samples. Fourteen Cronobacter serogroups have been identified. Among this collection of isolates, representatives of all but two $O$-antigen serotypes (O:5 and O:6) were recognized, consistently with several previous studies (Xu et al., 2014, 2015). C. sakazakii serotype O:1 was the dominant serotype, followed by serotype O:2. PCR-based $O$-antigen serotyping was reliable for characterizing isolates of E. coli and Listeria monocytogenes from clinical, food, and environmental samples (Singh and Manning, 2014; Chen et al., 2015). However, until recently there were only 17 defined serotypes across the whole Cronobacter genus (Mullane et al., 2008; Jarvis et al., 2011, 2013; Sun et al., 2012b), and the identification of unknown serotypes in this study indicated that an integrated standardized molecular serotyping protocol should be developed. Furthermore, there was also a contradiction in the specificity of the molecular serotyping classification presented by Jarvis et al. (2011), in which C. sakazakii O:3 and C. muytjensii O:1 were identified to be the same serogroup. Thus, further data on the distribution of serotypes (e.g., among clinical isolates) are needed to determine whether molecular serotyping is a useful alternative for epidemiological surveillance of Cronobacter.

Multilocus sequence typing is based on the sequences of gene fragments from a number of different housekeeping loci, and has also been used successfully to subtype Cronobacter isolates and to study the evolution and population genetics of various Cronobacter spp. (Joseph and Forsythe, 2012). MLST revealed 106 STs from the 171 Cronobacter isolates, of which 15 STs (ST1, ST4, ST7, ST8, ST13, ST23, ST40, ST60, ST64, ST93, ST158, ST233, ST264, ST281, and ST307) were associated with clinical isolates in different countries according to the PubMLST database (see footnote 1). ST4, ST7, ST8, and ST60 are particularly noteworthy. Cronobacter ST60 was the dominant ST in the current study. Unfortunately, one case of Cronobacter ST60 infection in an infant was detected at Wuhan Women and Children Medical Care Center Hospital (Wuhan City, China), and the pathogenesis mechanism of ST60 is still unclear (Cui et al., 2017). Therefore, we should increase our vigilance toward ST60 in raw vegetable as these can be a potential source of disease in children and the rest of the population; however, there is still not clear evidence of an epidemiological link between the consumption of raw vegetables and infection caused by Cronobacter. ST4 is the most dominant ST in clonal complex 4, which is regarded as a genetic signature for neonatal meningitis. To date, it is unclear why C. sakazakii clonal complex 4 predominates neonatal meningitis cases (Joseph and Forsythe, 2011; Hariri et al., 2013; Forsythe et al., 2014). Furthermore, seven C. malonaticus ST7 strains were also detected in this study. Considering that C. malonaticus ST7 is more often associated 


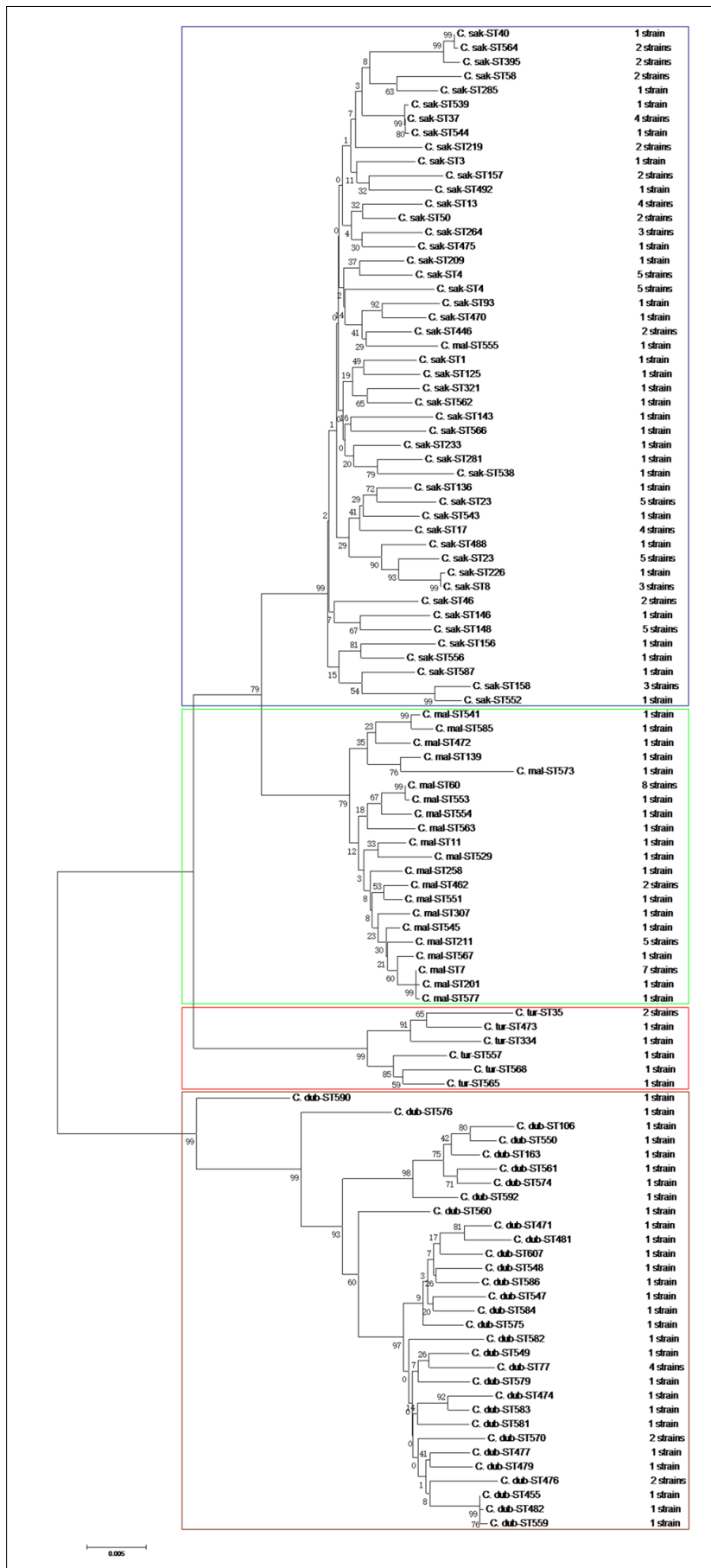

FIGURE 2 | Neighbor-joining phylogenetic tree based on the seven MLST loci (3,036 bp concatenated length) of Cronobacter spp. isolates. This tree was generated using MEGA (version 5.05) with 1,000 bootstrap replicates.

with adult infections than neonatal infections (Joseph and Forsythe, 2011; Alsonosi et al., 2015), and that ST7 is detected in uncooked vegetables served in ready-to-eat foods or salads, which adults eat more often than do neonates, raw vegetables may be a potential route of transmission and potential reservoirs for these infections. Three strains belonging to ST8 were also

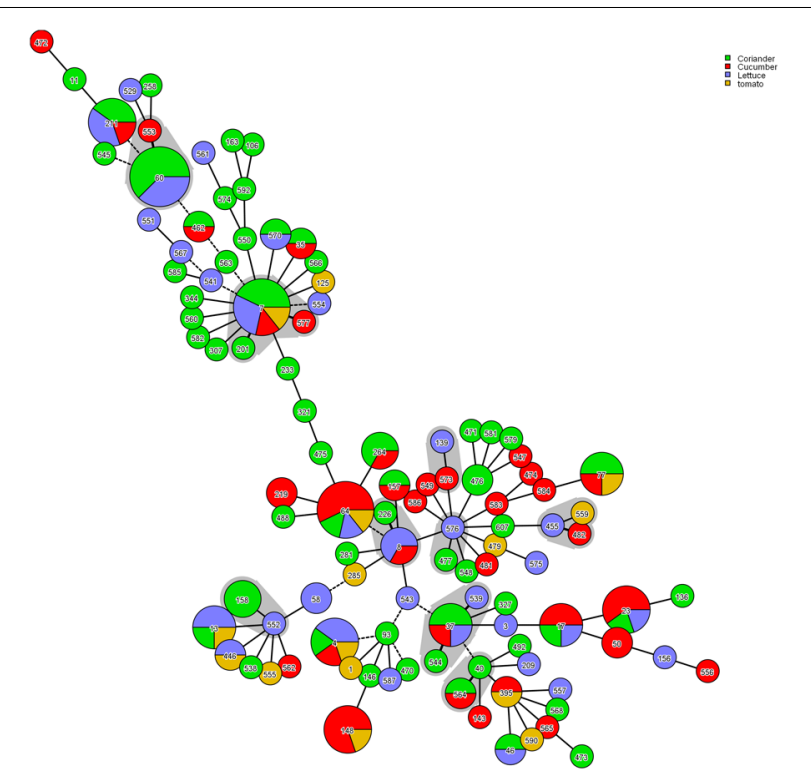

FIGURE 3 | Genetic relationships among the strains isolated in this study. A minimum spanning tree was constructed in BioNumerics software according to relationships among MLST alleles. Strains sharing four or more alleles are surrounding by a gray halo.

isolated. To date, although no ST8 strains have been shown to be associated with severe diseases, such as meningitis, but with diarrhea (Joseph and Forsythe, 2012), further studies of ST8 isolates are needed. Notably, most of these isolates harboring pathogenic STs tended to be located in South China. Surprisingly, up to 55 new STs were detected, indicating that further studies of the genetic populations of the Cronobacter genus are necessary to identify more STs, which could assist in risk identification and epidemiological studies of this pathogen.

According to molecular characterizations, there was high genetic diversity among the Cronobacter spp. in raw vegetables. MLST has high discriminatory power capable of showing genetic diversity among Cronobacter spp. isolates and can distinguish among the seven species using patterns of molecular markers. Furthermore, our study showed that the MLST patterns were generally associated with serotypes and provided a reliable prediction of the Cronobacter serovars. For instance, all C. malonaticus ST60 strains corresponded with the O:1 serotype. The association was not exclusive, however, as the seven isolated C. malonaticus ST7 strains corresponded with the O:2 serotype. The Cronobacter pathovar results agreed well with previous research (Ogrodzki and Forsythe, 2015). Continuous and comprehensive investigations should be carried out to improve our understanding of the genetic evolution of Cronobacter spp.

Because contaminated vegetables are considered transmission sources for human clinical isolates, the antimicrobial profiles of the isolates from raw vegetables were examined. All isolates were susceptible to AK, AMC, FEP, CIP, and IPM. However, some isolates exhibited high resistance to $\mathrm{KF}$ and intermediate resistance to $\mathrm{KF}$ and $\mathrm{KZ}$, which was consistent 
with other studies (Molloy et al., 2009; Chon et al., 2012). In addition, the chloramphenicol and gentamicin resistance rates were significantly different than those of Cronobacter spp. isolated from Chinese ready-to-eat foods in our laboratory (Xu et al., 2015) and various foods studied by Lee et al. (2012). The heterogeneity between vegetables and ready-to-eat foods suggested that the survival environment and source were vital for drug resistance. Notably, two isolates were multidrug-resistant strains (showing resistance to KF, SXT, and TE) in this study. These two multidrug-resistant isolates belonged to epidemiologically new STs, suggesting that these multidrug-resistant strains may have acquired resistance by vertical and horizontal gene transfer or from other microorganisms. Therefore, more attention is required while monitoring variations in the resistance patterns toward SXT and TE. In other studies, extended-spectrum $\beta$-lactamase-related genes (Girlich et al., 2001) and the colistin-resistance gene mcr-1 (Liu et al., 2017) were found in Cronobacter. AMP/CN or AMP/C usually serves as traditional therapy for Cronobacter spp. (Lai, 2001); therefore, intermediate resistance to AMP in Cronobacter isolates from raw vegetables may interfere with therapeutic approaches. Furthermore, some isolates exhibited intermediate resistance to CRO, TOB, and AMP. This could lead to the emergence of resistant strains under certain circumstances (Ruiz-Bolivar et al., 2011). Therefore, further studies are needed to determine the route of bacterial resistance and to moderate the use of drugs in order to prevent the emergence of antimicrobial resistance.

\section{CONCLUSION}

Overall, the findings of this study demonstrated a high contamination rate of Cronobacter in raw vegetable samples in China and suggested that raw vegetables may be potential carrier and transmission sources of Cronobacter. Based on MLST analyses, the pathogenic STs ST60, ST4, ST7, and ST8 were detected in raw vegetables; these STs would pose potential risks to both children and adults. In addition, all isolates were susceptible

\section{REFERENCES}

Alsonosi, A., Hariri, S., Kajsík, M., Oriešková, M., Hanulík, V., Röderová, M., et al. (2015). The speciation and genotyping of Cronobacter isolates from hospitalised patients. Eur. J. Clin. Microbiol. Infect. Dis. 34, 1979-1988. doi: 10.1007/s10096015-2440-8

Angelo, K. M., Chu, A., Anand, M., Nguyen, T. A., Bottichio, L., Wise, M., et al. (2015). Outbreak of Salmonella Newport infections linked to cucumbersUnited States, 2014. Morb. Mortal. Wkly. Rep. 64, 144-147.

Baldwin, A., Loughlin, M., Caubilla-Barron, J., Kucerova, E., Manning, G., Dowson, C., et al. (2009). Multilocus sequence typing of Cronobacter sakazakii and Cronobacter malonaticus reveals stable clonal structures with clinical significance which do not correlate with biotypes. BMC Microbiol. 9:223. doi: 10.1186/1471-2180-9-223

Blazkova, M., Javurkova, B., Vlach, J., Goselova, S., Karamonova, L., Ogrodzki, P., et al. (2015). Diversity of O-antigen designations within the genus Cronobacter: from Disorder to Order. Appl. Environ. Microbiol. 81, 5574-5582. doi: 10.1128/ AEM.00277-15

Cetinkaya, E., Joseph, S., Ayhan, K., and Forsythe, S. J. (2013). Comparison of methods for the microbiological identification and profiling of Cronobacter to AK, AMC, FEP, CIP, and IPM. However, strains showing resistance to $\mathrm{KF}$ and intermediate resistance to $\mathrm{KF}$ and $\mathrm{KZ}$ were found at a high ratio in raw vegetables. In this study, the high contamination rate and detection of pathogenic and new STs in raw vegetables indicated potential hazards to customers. To date, no standards for limitation of Cronobacter spp. in raw vegetables have been established in China. Thus, these findings highlight the need for dynamic screening related to transmission routes and the risk of food contamination of vegetables in China. However, the sample size was not large enough in this study, continuous surveillance of Cronobacter in China for reliable source tracking and determination of the contamination status is necessary.

\section{AUTHOR CONTRIBUTIONS}

$\mathrm{NL}, \mathrm{CL}$, and JZ contributed to the manuscript equally, done the article experiments and wrote the article together. QW gave the idea and experiments support. NL, JZ, and QW conceived the project. NL, CL, HZ, WH, YY, JW, YD, MC, WG, LX, and QY performed the experiments. JZ and QW supervised the project. NL and CL analyzed the data. NL wrote the article.

\section{ACKNOWLEDGMENTS}

We gratefully acknowledge the financial support of the National Natural Science Foundation of China (grant nos. 31371780, 31671951, and 31601571), the Science and Technology Planning Project of Guangdong Province, China (grant no. 2014A04040157), and the Science and Technology Planning Project of Guangzhou, China (grant no. 201508020037).

\section{SUPPLEMENTARY MATERIAL}

The Supplementary Material for this article can be found online at: https://www.frontiersin.org/articles/10.3389/fmicb. 2018.01149/full\#supplementary-material

species from ingredients used in the preparation of infant formula. Mol. Cell. Probes 27, 60-64. doi: 10.1016/j.mcp.2012.10.003

Chen, M., Wu, Q., Zhang, J., Wu, S., and Guo, W. (2015). Prevalence, enumeration, and pheno-and genotypic characteristics of Listeria monocytogenes isolated from raw foods in South China. Front. Microbiol. 6:1026. doi: 10.3389/fmicb. 2015.01026

Chen, W., Yang, J., You, C., and Liu, Z. (2016). Diversity of Cronobacter spp. isolates from the vegetables in the middle-east coastline of China. World J. Microbiol. Biotechnol. 32:90. doi: 10.1007/s11274-0162033-4

Chon, J. W., Song, K. Y., Kim, S. Y., Hyeon, J. Y., and Seo, K. H. (2012). Isolation and characterization of Cronobacter from desiccated foods in Korea. J. Food Sci. 77, M354-M358. doi: 10.1111/j.1750-3841.2012.02750.x

Clinical and Laboratory Standards Institute [CLSI] (2006). Methods for Antimicrobial Dilution and Disk Susceptibility Testing of Infrequently Isolated or Fastidious Bacteria. Approved Standard M45-A. Wayne, PA: Clinical and Laboratory Standards Institute.

Cui, J. H., Yu, B., Xiang, Y., Zhang, Z., Zhang, T., Zeng, Y. C., et al. (2017). Two cases of multi-antibiotic resistant Cronobacter spp. infections of infants in China. Biomed. Environ. Sci. 30, 601-605. doi: 10.3967/bes2017.079 
Depardieu, F., Podglajen, I., Leclercq, R., Collatz, E., and Courvalin, P. (2007). Modes and modulations of antibiotic resistance gene expression. Clin. Microbiol. Rev. 20, 79-114. doi: 10.1128/CMR.00015-06

Fei, P., Jiang, Y., Feng, J., Forsythe, S. J., Li, R., Zhou, Y., et al. (2017). Antibiotic and desiccation resistance of Cronobacter sakazakii and C. malonaticus isolates from powdered infant formula and processing environments. Front. Microbiol. 8:316. doi: 10.3389/fmicb.2017.00316

Forsythe, S. J., Dickins, B., and Jolley, K. A. (2014). Cronobacter, the emergent bacterial pathogen Enterobacter sakazakii comes of age; MLST and whole genome sequence analysis. BMC Genomics 15:1121. doi: 10.1186/1471-216415-1121

Girlich, D., Poirel, L., Leelaporn, A., Karim, A., Tribuddharat, C., Fennewald, M., et al. (2001). Molecular epidemiology of the integron-located VEB-1 extendedspectrum $\beta$-lactamase in nosocomial enterobacterial isolates in Bangkok, Thailand. J. Clin. Microbiol. 39, 175-182. doi: 10.1128/JCM.39.1.175-182.2001

Hariri, S., Joseph, S., and Forsythe, S. J. (2013). Cronobacter sakazakii ST4 strains and neonatal meningitis, United States. Emerg. Infect. Dis. 19, 175-177. doi: 10.3201/eid1901.120649

Holy, O., and Forsythe, S. (2014). Cronobacter spp. as emerging causes of healthcare-associated infection. J. Hosp. Infect. 86, 169-177. doi: 10.1016/j.jhin. 2013.09.011

Iversen, C., and Forsythe, S. (2003). Risk profile of Enterobacter sakazakii, an emergent pathogen associated with infant milk formula. Trends Food Sci. Technol. 14, 443-454. doi: 10.1016/S0924-2244(03)00155-9

Iversen, C., Mullane, N., McCardell, B., Tall, B. D., Lehner, A., Fanning, S., et al. (2008). Cronobacter gen. nov., a new genus to accommodate the biogroups of Enterobacter sakazakii, and proposal of Cronobacter sakazakii gen. nov., comb. nov., Cronobacter malonaticus sp. nov., Cronobacter turicensis sp. nov., Cronobacter muytjensii sp. nov., Cronobacter dublinensis sp. nov., Cronobacter genomospecies 1, and of three subspecies, Cronobacter dublinensis subsp. dublinensis subsp. nov., Cronobacter dublinensis subsp. lausannensis subsp. nov. and Cronobacter dublinensis subsp. lactaridi subsp. nov. Int. J. Syst. Evol. Microbiol. 58(Pt 6), 1442-1447. doi: 10.1099/ijs.0.65577-0

Jarvis, K. G., Grim, C. J., Franco, A. A., Gopinath, G., Sathyamoorthy, V., Hu, L., et al. (2011). Molecular characterization of Cronobacter lipopolysaccharide $\mathrm{O}$-antigen gene clusters and development of serotype-specific PCR assays. Appl. Environ. Microbiol. 77, 4017-4026. doi: 10.1128/AEM.00162-11

Jarvis, K. G., Yan, Q. Q., Grim, C. J., Power, K. A., Franco, A. A., Hu, L., et al. (2013). Identification and characterization of five new molecular serogroups of Cronobacter spp. Foodborne Pathog. Dis. 10, 343-352. doi: 10.1089/fpd.2012. 1344

Joseph, S., Cetinkaya, E., Drahovska, H., Levican, A., Figueras, M. J., and Forsythe, S. J. (2012a). Cronobacter condimenti sp. nov., isolated from spiced meat, and Cronobacter universalis sp. nov., a species designation for Cronobacter spp. genomospecies 1 , recovered from a leg infection, water and food ingredients. Int. J. Syst. Evol. Microbiol. 62(Pt 6), 1277-1283. doi: 10.1099/ijs.0.032292-0

Joseph, S., Desai, P., Ji, Y., Cummings, C. A., Shih, R., Degoricija, L., et al. (2012b). Comparative analysis of genome sequences covering the seven Cronobacter species. PLoS One 7:e49455. doi: 10.1371/journal.pone.0049455

Joseph, S., Sonbol, H., Hariri, S., Desai, P., McClelland, M., and Forsythe, S. J. (2012c). Diversity of the Cronobacter genus as revealed by multilocus sequence typing. J. Clin. Microbiol. 50, 3031-3039. doi: 10.1128/jcm.00905-12

Joseph, S., and Forsythe, S. J. (2011). Predominance of Cronobacter sakazakii sequence type 4 in neonatal infections. Emerg. Infect. Dis. 17, 1713-1715. doi: $10.3201 /$ eid 1709.110260

Joseph, S., and Forsythe, S. J. (2012). Insights into the emergent bacterial pathogen Cronobacter spp., generated by multilocus sequence typing and analysis. Front. Microbiol. 3:397. doi: 10.3389/fmicb.2012.00397

Lai, K. K. (2001). Enterobacter sakazakii infections among neonates, infants, children, and adults - Case reports and a review of the literature. Medicine 80, 113-122. doi: 10.1097/00005792-200103000-00004

Lee, Y. D., Park, J. H., and Chang, H. (2012). Detection, antibiotic susceptibility and biofilm formation of Cronobacter spp. from various foods in Korea. Food Control 24, 225-230. doi: 10.1016/j.foodcont.2011.09.023

Li, Z., Ge, W., Li, K., Gan, J., Zhang, Y., Zhang, Q., et al. (2016). Prevalence and characterization of Cronobacter sakazakii in retail milk-based infant and baby foods in Shaanxi, China. Foodborne Pathog. Dis. 13, 221-227. doi: 10.1089/fpd. 2015.2074
Liu, B.-T., Song, F.-J., Zou, M., Hao, Z.-H., and Shan, H. (2017). Emergence of colistin resistance gene mcr-1 in Cronobacter sakazakii producing NDM-9 and in Escherichia coli from the same animal. Antimicrob. Agents Chemother. 61:e01444-16. doi: 10.1128/AAC.01444-16

Molloy, C., Cagney, C., O’Brien, S., Iversen, C., Fanning, S., and Duffy, G. (2009). Surveillance and characterisation by pulsed-field gel electrophoresis of Cronobacter spp. in farming and domestic environments, food production animals and retail foods. Int. J. Food Microbiol. 136, 198-203. doi: 10.1016/j. ijfoodmicro.2009.07.007

Mullane, N., O'Gaora, P., Nally, J. E., Iversen, C., Whyte, P., Wall, P. G., et al. (2008). Molecular analysis of the Enterobacter sakazakii O-antigen gene locus. Appl. Environ. Microbiol. 74, 3783-3794. doi: 10.1128/AEM.02302-07

National Standard of the People's Republic of China (2010). National Food Safety Standard Food Microbiological Examination: Enterobacter sakazakii (GB 4789.40-2010). Beijing: Ministry of Health of People's Republic of China.

National Standard of the People's Republic of China (2013). National Food Safety Standard Food Microbiological Examination: Vibrio parahaemolyticus (GB 4789.7-2013). Beijing: Ministry of Health of People's Republic of China.

Nygård, K., Lassen, J., Vold, L., Andersson, Y., Fisher, I., Löfdahl, S., et al. (2008). Outbreak of Salmonella Thompson infections linked to imported rucola lettuce. Foodborne Pathog. Dis. 5, 165-173. doi: 10.1089/fpd.2007.0053

Ogrodzki, P., and Forsythe, S. (2015). Capsular profiling of the Cronobacter genus and the association of specific Cronobacter sakazakii and C. malonaticus capsule types with neonatal meningitis and necrotizing enterocolitis. BMC Genomics 16:758. doi: 10.1186/s12864-015-1960-z

Patrick, M. E., Mahon, B. E., Greene, S. A., Rounds, J., Cronquist, A., Wymore, K., et al. (2014). Incidence of Cronobacter spp. infections, United States, 2003-2009. Emerg. Infect. Dis. 20, 1520-1523. doi: 10.3201/eid2009.140545

Ruiz-Bolivar, Z., Neuque-Rico, M. C., Poutou-Pinales, R. A., Carrascal-Camacho, A. K., and Mattar, S. (2011). Antimicrobial susceptibility of Listeria monocytogenes food isolates from different cities in Colombia. Foodborne Pathog. Dis. 8, 913-919. doi: 10.1089/fpd.2010.0813

Schmid, M., Iversen, C., Gontia, I., Stephan, R., Hofmann, A., Hartmann, A., et al. (2009). Evidence for a plant-associated natural habitat for Cronobacter spp. Res. Microbiol. 160, 608-614. doi: 10.1016/j.resmic.2009.08.013

Singh, N., Goel, G., and Raghav, M. (2015). Prevalence and characterization of Cronobacter spp. from various foods, medicinal plants, and environmental samples. Curr. Microbiol. 71, 31-38. doi: 10.1007/s00284-015-0816-8

Singh, P., and Manning, S. D. (2014). "Molecular characterization of Shiga toxinproducing Escherichia coli," in DNA Methods in Food Safety: Molecular Typing of Foodborne and Waterborne Bacterial Pathogens, ed. O. A. Oyarzabal (Hoboken, NJ: John Wiley \& Sons), 275-302.

Söderström, A., Osterberg, P., Lindqvist, A., Jönsson, B., Lindberg, A., Blide, U. S., et al. (2008). A large Escherichia coli O157 outbreak in Sweden associated with locally produced lettuce. Foodborne Pathog. Dis. 5, 339-349. doi: 10.1089/fpd. 2007.0065

Stoll, B. J., Hansen, N., Fanaroff, A. A., and Lemons, J. A. (2004). Enterobacter sakazakii is a rare cause of neonatal septicemia or meningitis in VLBW infants. J. Pediatr. 144, 821-823.

Sulovska, K., Fiserova, E., Chvostekova, M., and Adamek, M. (2017). Appropriateness of gait analysis for biometrics: initial study using FDA method. Measurement 105, 1-10. doi: 10.1016/j.measurement.2017.03.042

Sun, Y., Arbatsky, N. P., Wang, M., Shashkov, A. S., Liu, B., Wang, L., et al. (2012a). Structure and genetics of the O-antigen of Cronobacter turicensis G3882 from a new serotype, C. turicensis $\mathrm{O} 2$, and identification of a serotype-specific gene. FEMS Immunol. Med. Microbiol. 66, 323-333. doi: 10.1111/j.1574-695X.2012. 01013.x

Sun, Y., Wang, M., Wang, Q., Cao, B., He, X., Li, K., et al. (2012b). Genetic analysis of the Cronobacter sakazakii $\mathrm{O} 4$ to $\mathrm{O} 7 \mathrm{O}$-antigen gene clusters and development of a PCR assay for identification of all C. sakazakii O serotypes. Appl. Environ. Microbiol. 78, 3966-3974. doi: 10.1128/AEM.07825-11

Sun, Y., Wang, M., Liu, H., Wang, J., He, X., Zeng, J., et al. (2011). Development of an O-antigen serotyping scheme for Cronobacter sakazakii. Appl. Environ. Microbiol. 77, 2209-2214. doi: 10.1128/AEM.02229-10

Taylor, E. V., Nguyen, T. A., Machesky, K. D., Koch, E., Sotir, M. J., Bohm, S. R., et al. (2013). Multistate outbreak of Escherichia coli O145 infections associated with romaine lettuce consumption, 2010. J. Food Prot. 76, 939-944. doi: 10.4315/0362-028X.JFP-12-503 
Van Acker, J., de Smet, F., Muyldermans, G., Bougatef, A., Naessens, A., and Lauwers, S. (2001). Outbreak of necrotizing enterocolitis associated with Enterobacter sakazakii in powdered milk formula. J. Clin. Microbiol. 39, 293-297. doi: 10.1128/JCM.39.1.293-297.2001

Tuffs, A. (2011). Outbreak of $E$ coli in Germany is linked to cucumbers from Spain. BMJ 342:1230. doi: 10.1136/bmj.d3394

Vojkovska, H., Karpiskova, R., Orieskova, M., and Drahovska, H. (2016). Characterization of Cronobacter spp. isolated from food of plant origin and environmental samples collected from farms and from supermarkets in the Czech Republic. Int. J. Food Microbiol. 217, 130-136. doi: 10.1016/j.ijfoodmicro. 2015.10.017

World Health Organization [WHO] (2007). Critically Important Antimicrobials for Human Medicine: Categorization for the Development of Risk Management Strategies to Contain Antimicrobial Resistance Due to Non-Human Antimicrobial Use: Report of the Second Who Expert Meeting, Copenhagen, 29-31 May. Geneva: World Health Organization.

Xu, X., Li, C., Wu, Q., Zhang, J., Huang, J., and Yang, G. (2015). Prevalence, molecular characterization, and antibiotic susceptibility of Cronobacter spp. in Chinese ready-to-eat foods. Int. J. Food Microbiol. 204, 17-23. doi: 10.1016/j. ijfoodmicro.2015.03.003

Xu, X., Wu, Q., Zhang, J., Ye, Y., Yang, X., and Dong, X. (2014). Occurrence and characterization of Cronobacter spp. in powdered formula from Chinese retail markets. Foodborne Pathog. Dis. 11, 307-312. doi: 10.1089/fpd.2013.1657

Ye, Y., Li, H., Wu, Q., Chen, M., Lu, Y., and Yan, C. (2014a). Isolation and phenotypic characterization of Cronobacter from dried edible macrofungi samples. J. Food Sci. 79, M1382-M1386. doi: 10.1111/1750-3841.12513
Ye, Y., Li, H., Wu, Q., Zhang, J., and Lu, Y. (2014b). The Cronobacter sp. in milk and dairy products: detection and typing. Int. J. Dairy Technol. 67, 167-175. doi: 10.1111/1471-0307.12111

Ye, Y., Wu, Q., Xu, X., Yang, X., Dong, X., and Zhang, J. (2010). The phenotypic and genotypic characterization of Enterobacter sakazakii strains from infant formula milk. J. Dairy Sci. 93, 2315-2320. doi: 10.3168/jds.20092662

Ye, Y., Wu, Q., Yao, L., Dong, X., Wu, K., and Zhang, J. (2009). Analysis of a consensus fragment in ERIC-PCR fingerprinting of Enterobacter sakazakii. Int. J. Food Microbiol. 132, 172-175. doi: 10.1016/j.ijfoodmicro.2009. 03.018

Zhang, H., Marsh, T., and Mccluskey, J. (2009). An event analysis of the 2006 E. coli outbreak in spinach and lettuce. J. Agric. Resour. Econ. 34, $553-553$.

Conflict of Interest Statement: The authors declare that the research was conducted in the absence of any commercial or financial relationships that could be construed as a potential conflict of interest.

Copyright (C) 2018 Ling, Li, Zhang, Wu, Zeng, He, Ye, Wang, Ding, Chen, Xue, Ye and Guo. This is an open-access article distributed under the terms of the Creative Commons Attribution License (CC BY). The use, distribution or reproduction in other forums is permitted, provided the original author(s) and the copyright owner are credited and that the original publication in this journal is cited, in accordance with accepted academic practice. No use, distribution or reproduction is permitted which does not comply with these terms. 